\title{
A Unified Geometric Rule for Designing Nanomagnetism in Graphene
}

\author{
Decai Yu', Elizabeth M. Lupton ${ }^{1}$, H. J. Gao ${ }^{2}$, Chao Zhang ${ }^{3}$, and Feng Liu ${ }^{1}(\bowtie)$ \\ ${ }^{1}$ Department of Materials Science and Engineering, University of Utah, Salt Lake City, UT 84112, USA \\ ${ }^{2}$ Institute of Physics, Chinese Academy of Science, Beijing 100080, China \\ ${ }^{3}$ School of Engineering Physics, University of Wollongong, NSW 2522, Australia \\ Received: 30 September 2008 / Revised: 4 November 2008 / Accepted: 14 November 2008 \\ (C)Tsinghua Press and Springer-Verlag 2008. This article is published with open access at Springerlink.com
}

\begin{abstract}
Based on the underlying graphene lattice symmetry and an itinerant magnetism model on a bipartite lattice, we propose a unified geometric rule for designing graphene-based magnetic nanostructures: spins are parallel (ferromagnetic (FM)) on all zigzag edges which are at angles of $0^{\circ}$ and $120^{\circ}$ to each other, and antiparallel (antiferromagnetic (AF)) at angles of $60^{\circ}$ and $180^{\circ}$. The rule is found to be consistent with all the systems that have been studied so far. Applying the rule, we predict several novel graphene-based magnetic nanostructures: 0-D FM nanodots with the highest possible magnetic moments, 1-D FM nanoribbons, and 2-D magnetic superlattices.
\end{abstract}

\section{KEYWORDS}

Nanomagnetism, graphene-based nanostructures, magnetic ordering

The electronic properties of crystalline structures are closely related to their underlying lattice symmetries. Understanding such structure-property relationships is of great scientific interest and provides the knowledge base for technological development. The complex electronic properties are often governed by simple geometric rules. One famous example is the relationship between the electronic properties of carbon nanotubes (CNTs) and their chirality [1, 2]. Using $(m, n)$ to denote the chirality, a CNT is metallic if $(m-n)$ is divisible by 3 and semiconducting otherwise. This rule has been proven to be amazingly useful in understanding the electronic properties of CNTs. Similar rules have been discussed for graphene nanoribbons with modifications in respect to their edge states [3-5].
Recently, various graphene-based nanostructures (GBNs), such as graphene nanoribbons [6-10], nanodots [11-14], and nanoholes [15], with zigzag edges have been shown to exhibit magnetism, making them an interesting new class of organic nanomagnets. The magnetization in GBNs originates from the localized edge states [3-5] that give rise to a high density of states at the Fermi level, rendering a spin-polarization instability [16, 17]. However, the energies of different magnetic phases (e.g., ferromagnetic (FM) vs antiferromagnetic (AF)) of a GBN can only be determined as after-math post priori first principles calculations. Either the FM or AF phase may be the ground state depending on the underlying GBN symmetries. There has been a

Address correspondence to fliu@eng.utah.edu 
lack of a unified guiding principle in designing the rich variety of possible magnetic nanostructures in graphene. Here, we propose a generic geometric rule that underlies the magnetic ordering of all the GBNs.

It has been shown that the ground state magnetic ordering within a single nanoribbon [6-10], nanodot [11-14], or nanohole [15] is consistent with the theorem of itinerant magnetism in a bipartite lattice within the one-orbital Hubbard model [18]. Graphene consists of two atomic sublattices (A and $\mathrm{B})$, and a zigzag edge must be either on an A- or B-lattice. It is found that in a given GBN, two edges will be FM-coupled if they are on the same sublattice and AF-coupled if they are not. The total spin $S$ of the ground state equals $1 / 2\left|N_{\mathrm{B}}-N_{\mathrm{A}}\right|$, where $N_{\mathrm{B}}\left(N_{\mathrm{A}}\right)$ is the number of atoms on the $\mathrm{B}(\mathrm{A})$ sublattice. These observations indicate that there exist a set of rules to define the condition of magnetism in graphene [11, 15]. Furthermore, by simply examining the graphene lattice symmetry, we formulate a generic "geometric" rule that dictates the edge types in a GBN for its given symmetry, so as to define its magnetic order. Applying this simple geometric rule, we design a series of novel magnetic GBNs whose rule-defined ground states are further confirmed by first principles calculations.

The basic principle of the geometric rule is illustrated in Fig. 1. Because of the underlying honeycomb lattice symmetry, the relationship between any two zigzag edges is uniquely defined by their relative angle to each other. Specifically, atoms on the same zigzag edge belong to the same sublattice (either A-lattice (red)) or B-lattice (blue)); atoms on two different zigzag edges belong to the same sublattice if the two edges are at an angle of $0^{\circ}$ or $120^{\circ}$ to each other, but different sublattices if at an angle of $60^{\circ}$ or $180^{\circ}$ to each other. To avoid confusion, the angle between any two edges is defined formally as the angle between the two normal vectors of the edges. Then, the unified design rule states: two zigzag edges are FM-coupled if they are at an angle of $0^{\circ}$ or $120^{\circ}$ and AF-coupled if at an angle of $60^{\circ}$ or $180^{\circ}$. The rule partially reflects the three-fold rotational symmetry of the graphene honeycomb lattice and the reflection symmetry between the two sublattices. Below, we elaborate on how this rule

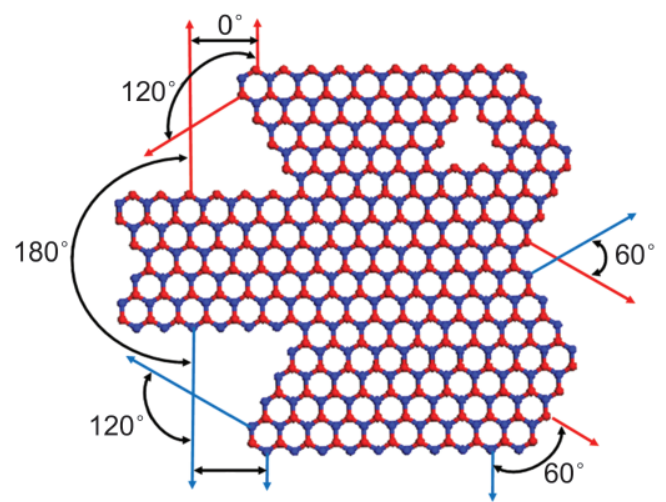

Figure 1 Schematic illustration of the underlying geometric relationship between zigzag edges in graphene. It illustrates that the edges are on the same sublattice (A (red) or B (blue)) if they are at an angle of $0^{\circ}$ or $120^{\circ}$ to each other, but on different sublattices (A vs B) if at an angle of $60^{\circ}$ or $180^{\circ}$

can be applied in designing three different classes of magnetic GBNs: the 0-D nanodots, 1-D nanoribbons, and 2-D nanohole superlattices.

Suppose we cut the graphene into small 0-D nanodots bounded by zigzag edges, as shown in Fig. 2. According to the rule, a triangular dot is FM (Fig. 2(a)), because all three edges are at $120^{\circ}$ to each other; a hexagonal (also true for a rhombus shaped) dot is $\mathrm{AF}$, because any two neighboring edges are at $60^{\circ}$ to each other. The magnetic order is graphically shown in Fig. 2 with the color coding of the edge, i.e. the red A-edge (let's say spin up) vs the blue B-edge (spin down). The same color coding will be used throughout the paper. The magnetic ground states of the nanodots predicted by this simple rule are found to be consistent with the existing first principles calculations of all different shapes of nanodots [11-14]. Also, the same is true for individual nanoholes (antidots) punched in graphene [15] (see Fig. 4 and related discussion below).

Only FM nanodots have a net magnetic moment, while AF nanodots have zero moment. For magnetic and spintronic applications, it is desirable to search for FM nanodots with a net moment as high as possible. This search would be rather difficult with time consuming first principles calculations. With the aid of a generic design rule, such searches become much easier. As the rule suggests, the first key to the design is to eliminate edges which are at $60^{\circ}$ or $180^{\circ}$ to each other, so that the nanodots contain only edges which are at $0^{\circ}$ or $120^{\circ}$ to each other and they all have the same spin orientation. The second key is to elongate the edge length as much as possible to 


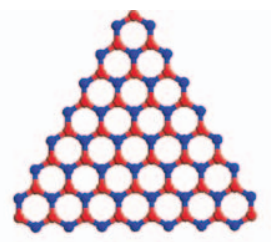

(a)

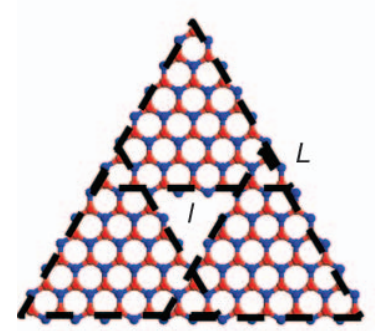

(c)

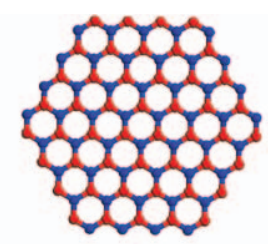

(b)

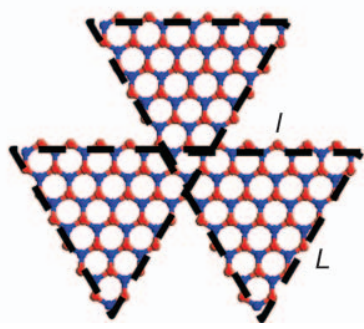

(d)
Figure 2 Schematics of graphene nanodots showing the different zigzag edge configurations defined by the underlying geometry: (a) a triangular nanodot bounded by one type of edge belonging to the same sublattice, the B-lattice (blue); (b) a hexagonal nanodot bounded by two types of edges alternating around the dot; (c) a nanodot formed by stacking three up-triangles together sharing a common edge (or viewed as a larger up-triangule embedded with a smaller down-triangule); (d) same as (c) with three down-triangles stacked together sharing a common corner

maximize the total net moment.

Figures 2(c) and 2(d) illustrate two elemental designs which fulfill these two key requirements. The FM nanodot in Fig. 2(c) is derived from Fig. 2(a) by punching a small down-triangle inside a larger uptriangle (or conversely a small up-triangle inside a larger down-triangle) to make all edge B-type (blue). The FM nanodot in Fig. 2(d) is derived from Fig. 2(b) by cutting each of three B-type edges (blue) in the hexagon into two A-type edges (red) (or conversely cutting three A-type into six B-type), so that all the edges are of A-type.

Another way to think about designing FM nanodots with high moments is by stacking many triangular FM dots together. Then, as indicated by the dashed-line triangles, one can view Fig. 2(c) as one way of stacking triangular dots together by sharing their edges, and Fig. 2(d) as another way of stacking triangular dots together by sharing their corners. By exploiting these two design elements, FM nanodots with the largest total magnetic moments can be created.

Next, we discuss the design of 1-D nanoribbons as shown in Fig. 3. The simplest ribbon structure is the one with two straight edges. According to the rule, the two edges are $\mathrm{AF}$ coupled because they are at $180^{\circ}$ to each other (Fig. 3(a)), which is wellestablished by first principles calculations [6-9]. Also, the sawtooth-like ribbons with parallel edges are FM [10]. The AF nanoribbons can be useful in their own right. For example, under a transverse electrical field, they become an interesting class of semimetal [7]. In other applications, FM nanoribbons may be desirable. Previously, researchers have proposed the idea of converting the AF nanoribbons into FM ones by extrinsic effects such as introducing defects and impurity atoms/molecules [9] along one of the two edges. Here, by applying the geometric rule, we design intrinsic (pure carbon) FM nanoribbons by manipulating their edge geometries.

The trick is to change the relative orientations of the two edges so that they become at $0^{\circ}$ or $120^{\circ}$ to each other instead of at $180^{\circ}$ as in the straight ribbons. Figures 3(b) and 3(c) show two such designs of FM ribbons. In Fig. 3(b), we maintain one straight edge of the ribbon while cutting the other edge into a sawtooth shape with $60^{\circ}$ contact angle. As such, we make a tree-saw shaped FM nanoribbon. In Fig. 3(c), we cut both edges into another kind of saw-tooth shape to make a Christmas-tree shaped FM nanoribbon.

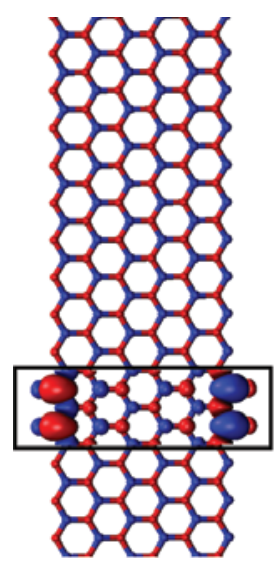

(a)

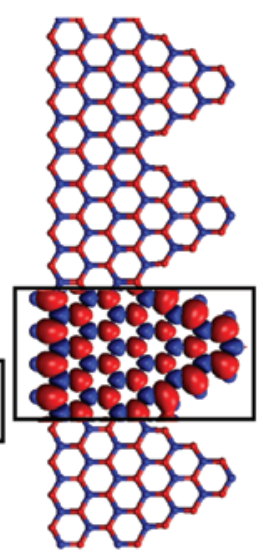

(b)

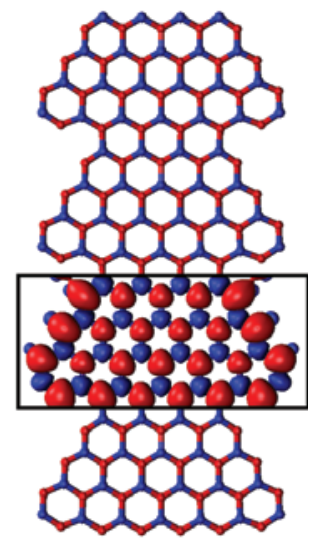

(c)
Figure 3 Schematics of nanoribbons with different edge configurations: (a) An AF nanoribbon with straight edges; (b) an FM tree-saw nanoribbon; (c) an FM Christmas-tree nanoribbon. The rectangles indicate one unit cell for each ribbon, which show the calculated spin density contour of the ground state magnetic configuration

We have performed first principles calculations which have indeed confirmed that these nanoribbons

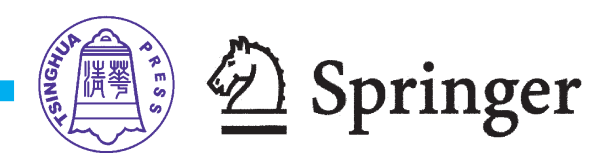


have an FM ground state. Our calculations were performed using the pseudopotential plane-wave method within the spin-polarized generalized gradient approximation as before $[9,15]$. We used a rhombus supercell with a vacuum layer of $\sim 10 \AA$ to separate the graphene planes and a plane wave cut-off of $22.1 \mathrm{Rd}$. All the carbon atoms on the edge with dangling bonds are terminated by hydrogen atoms. The system is relaxed until the force on each atom is minimized to less than $0.01 \mathrm{eV} / \AA$.

The ground state spin densities within one unit cell of nanoribbon are plotted in Fig. 3 to illustrate their magnetic ordering (see density contours inside the rectangular unit cells). For the tree-saw nanoribbon in Fig. 3(b), the FM ground state is found to be $100 \mathrm{meV}$ per unit cell lower than the AF state which is lower than the paramagnetic (PM) state by $220 \mathrm{meV}$. For the Christmas-tree nanoribbon in Fig. $3(\mathrm{c})$, the FM ground state is found to be $33 \mathrm{meV}$ per unit cell lower than the PM state which is lower than the AF state by $8 \mathrm{meV}$. The total magnetic moment in the FM ground state is calculated to be 3.0 and 2.0 $\mu_{\mathrm{B}}$ per unit cell for the tree-saw and Christmas-tree nanoribbon, respectively, which are equal to $\left(N_{\mathrm{B}}-N_{\mathrm{A}}\right)$ as predicted from the itinerant magnetism model in a bipartite lattice [18].

Lastly, we apply the design rule to a new class of 2-D magnetic GBNs, the graphene nanohole (NH) superlattices [15], as shown in Fig. 4. Suppose a periodic array of nano-sized holes with zigzag edges are punched in graphene. Each individual $\mathrm{NH}$, which is essentially an inverse structure of a nanodot (anti-dot), has the same magnetic configuration as a nanodot [15]. Then, according to the design rule, to construct a superlattice the triangular FM NH will be the natural choice with non-zero net moment, and can be viewed effectively as a "super magnetic atom" in the superlattice. To increase the moment of such super magnetic atoms, more complicated $\mathrm{NH}$ geometries like the inverse structures of Figs. 2(b) and 2(c) can also be designed.

In designing a magnetic NH superlattice, the generic geometric rule can be applied not only to the intra-NH spin ordering within each $\mathrm{NH}$, but also to the inter-NH spin ordering among different NHs. Two triangular NHs will be FM-coupled if their corresponding edges are at $0^{\circ}$ and $120^{\circ}$ to each other, but AF-coupled if their corresponding edges are at $60^{\circ}$ and $180^{\circ}$ to each other. Therefore, an overall FM superlattice can be designed using a periodic repeating unit cell containing one triangular $\mathrm{NH}$ as shown in Fig. 4(a), while an AF superlattice can be obtained by using a unit cell containing two antiparalleled triangular NHs (one up- and one downtriangle) as shown in Fig. 4(b).

The ground state spin densities within one unit cell, as obtained from first principles calculations, are plotted in Figs. 4(a) and 4(b) to confirm their respective FM and AF ordering as predicted by the rule. For Fig. 4(a), the FM ground state is found to be $61.3 \mathrm{meV}$ per unit cell lower than the AF state which is lower than the paramagnetic (PM) state by $11.8 \mathrm{meV}$. For Fig. 4(b), the AF ground state is found to be $63.0 \mathrm{meV}$ per unit cell lower than the FM state which is lower than the PM state by $23.7 \mathrm{meV}$. The calculated total magnetic moments for the FM (Fig. 4(a)) and AF (Fig. 4(b)) ground states are $2.0 \mu_{\mathrm{B}}$ and 0.0 $\mu_{\mathrm{B}}$ per unit cell, respectively, which are again equal to $\left(N_{\mathrm{B}}-N_{\mathrm{A}}\right)$ as predicted from the itinerant magnetism model in a bipartite lattice [18].

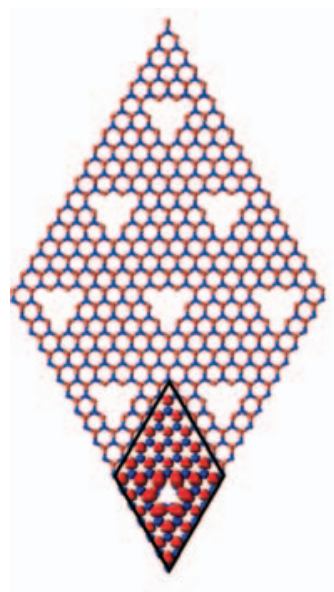

(a)

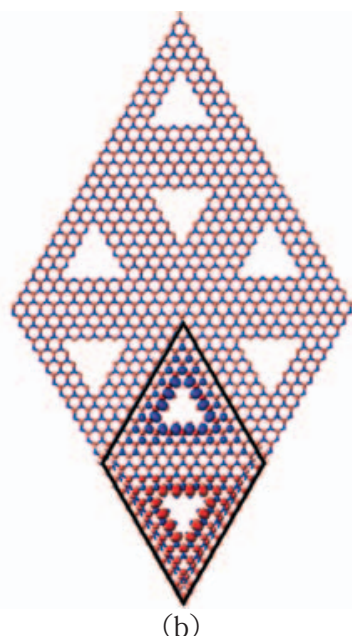

(b)
Figure 4 Schematics of the graphene nanohole superlattice: (a) an FM hexagonal NH lattice; (b) an AF hexagonal NH lattice. The rhombuses indicate one unit cell of each superlattice, and show the calculated spin density contour of the ground state magnetic configuration

In view of the successful application of the rule in designing nanomagnetic graphene for various dimensions as discussed above, we now comment briefly on the physical origin underlying the rule. The 
magnetic couplings may be attributed to two distinct mechanisms: one is the coupling of nonbonding states, which arises from topological constraints; the second is the magnetic instability of low energy states, which could be present when the size of the pattern is sufficiently large. For the first mechanism, size is not an issue and either FM or AF coupling could be possible for short zigzag edges that are only a couple of benzene rings long. The nature of the electron-electron interactions seem to dictate the FM coupling between the same non-bonding edges (A or B) and AF coupling between different edges (A vs B). However, when nonbonding states are not present and the size of the system is small, such as the hexagonal nanodot in Fig. 2(b), the edges will not be spin polarized and the geometric rule therefore does not hold. This is indeed confirmed by our firstprinciples calculations which showed a PM ground state for very small hexagonal nanoholes or two small triangular nanoholes of opposite orientation (see Fig. 4(b)) very close to each other [15].

In conclusion, we propose a generic geometric rule for designing the magnetic ground state of GBNs bounded by zigzag edges, by unifying the underlying graphene lattice symmetry with an itinerant magnetism model on a bipartite lattice. The rule predicts that any two zigzag edges will be FM-coupled if they are at an angle of $0^{\circ}$ or $120^{\circ}$ and AF-coupled if at an angle of $60^{\circ}$ or $180^{\circ}$. We have applied the rule to design a series of novel 0-D, 1-D and 2-D GBNs, and confirmed the predictions by first principles calculations. We expect this rule to provide a useful guiding principle in future studies of nanomagnetism in graphene and related materials, such as nanopatterned graphite.

\section{Acknowledgements}

The work at Utah is supported by DOE; the work at IOP is supported by NSFC; the work at UoW is supported by the Australian Research Council (ARC) Discovery grant. Liu also acknowledges support by an ARC international professorial fellowship and Dr. X.L. Wang for helping to prepare the figures. First principles calculations were performed on computers at DOE-NERSC and CHPC of University of Utah.

\section{References}

[1] Saito, R.; Fujita, M.; Dresselhaus, G.; Dresselhaus, M. S. Electronic structure of chiral graphene tubules. Appl. Phys. Lett. 1992, 60, 2204-2206.

[2] Saito, R.; Dresselhaus, G.; Dresselhaus, M. S. Electronic structure of double-layer graphene tubules. J. Appl. Phys. 1993, 73, 494-500.

[3] Nakada, K.; Fujita, M.; Dresselhaus, G.; Dresselhaus, M. S. Edge state in graphene ribbons: Nanometer size effect and edge shape dependence. Phys. Rev. B 1996, 54, 17954-17961.

[4] Fujita, M.; Wakabayashi, K.; Nakada, K.; Kusakabe, K. Peculiar localized state at zigzag graphite edge. J. Phys. Soc. Jpn. 1996, 65, 1920-1923.

[5] Yan, Q. M.; Huang, B.; Yu, J.; Zheng, F. W.; Zang, J.; Wu, J.; Gu, B. L.; Liu, F.; Duan, W. H. Intrinsic current-voltage characteristics of graphene nanoribbon transistors and effect of edge doping. Nano Lett. 2007, 7, 1469-1473.

[6] Kusakabe, K.; Maruyama, M. Magnetic nanographite. Phys. Rev. B 2003, 67, 092406.

[7] Son, Y. W.; Cohen, M. L.; Louie, S. G. Half-metallic graphene nanoribbons. Nature 2006, 444, 347-342.

[8] Pisani, L.; Chan, J. A.; Montanari, B.; Harrison, N. M. Electronic structure and magnetic properties of graphitic ribbons. Phys. Rev. B 2007, 75, 064418.

[9] Huang, B.; Liu, F.; Wu, J.; Gu, B. L.; Duan, W. H. Suppression of spin polarization in graphene nanoribbons by edge defects and impurities. Phys. Rev. B 2008, 77, 153411.

[10] Wu, X. J.; Zeng, X. C. Sawtooth-like graphene nanoribbon. Nano Res. 2008, 1, 40-45.

[11] Fernandez-Rossier, J.; Palacios, J. J. Magnetism in graphene nanoislands. Phys. Rev. Lett. 2007, 99, 177204.

[12] Wang, W. L.; Meng, S.; Kairas, E. Graphene nanoflakes with large spin. Nano Lett. 2007, 8, 241-245.

[13] Ezawa, M. Metallic graphene nanodisks: Electronic and magnetic properties. Phys. Rev. B 2007, 76, 245415.

[14] Hod, O.; Barone, V.; Scuseria, G. E. Half-metallic graphene nanodots: A comprehensive first principles theoretical study. Phys. Rev. B 2008, 77, 035411.

[15] Yu, D. C.; Lupton, E. M.; Liu, M.; Liu, W.; Liu, F. Collective magnetic behavior of graphene nanohole superlattices. Nano Res. 2008, 1, 56-62.

[16] Slater, J. C. The ferromagnetism of nickel. Phys. Rev. 1936, 49, 537-545.

[17] Liu, F.; Khanna, S. N.; Jena, P. Quantum size effect on the magnetism of finite systems. Phys. Rev. B 1990, 42, 976-979.

[18] Lieb, E. H. Two theorems on the Hubbard model. Phys. Rev. Lett. 1989, 62, 1201-1204

\section{国备 Springer}

